\title{
THE TOPOLOGICAL DEGREE AND GALERKIN APPROXIMATIONS FOR NONCOMPACT OPERATORS IN BANACH SPACES
}

\author{
BY FELIX E. BROWDER AND W. V. PETRYSHYN
}

Communicated by Gian-Carlo Rota, March 11, 1968

Let $X$ and $Y$ be real Banach spaces, $G$ a bounded open subset of $X$, $\operatorname{cl}(G)$ its closure in $X$, bdry $(G)$ its boundary in $X$. We consider mappings $T$, (nonlinear, in general), of $\operatorname{cl}(G)$ into $Y$ which are $A$-proper, in the sense defined below, with respect to a given approximation scheme of generalized Galerkin type. We define a generalized concept of topological degree for such mappings with respect to the given approximation scheme, and show that this degree (which may be multivalued) has the basic properties of the classical Leray-Schauder degree (where the latter is defined on the narrower class of maps of $X$ into $X$ of the form $I+C$, with $I$ the identity and $C$ compact).

For a wide class of $A$-proper mappings $T$ of the form $T=H+C$, with $H$ an $A$-proper homeomorphism of a suitable type and $C$ compact, we show that the degree is single-valued and coincides with another generalized degree studied in Browder [9] and BrowderNussbaum [11], and in particular is independent of the approximation scheme involved. In particular, this holds if $H$ is strongly accretive from $X$ to $X$ (cf. Browder [4], [5], [6], [8]), including as a very special case all maps $H$ of the form $H=I-U$, with $U$ a strict contraction.

Definjtion 1. Let $X$ and $Y$ be Banach spaces. By an (oriented) approximation scheme for mappings from $X$ to $Y$, we mean: an increasing sequence $\left\{X_{n}\right\}$ of oriented finite dimensional subspaces of $X$, an increasing sequence $\left\{Y_{n}\right\}$ of oriented finite dimensional subspaces of $Y$, and a sequence of linear projection maps $\left\{Q_{n}\right\}$ with $Q_{n}$ mapping $Y$ on $Y_{n}$ such that $\operatorname{dim}\left(X_{n}\right)=\operatorname{dim}\left(Y_{n}\right)$ for all $n, \bigcup_{n} X_{n}$ is dense in $X$, and $Q_{n} y \rightarrow y$ as $n \rightarrow \infty$ for all $y$ in $Y$.

DefINITION 2. Let $G$ be a bounded open subset of $X, T$ a mapping of $\operatorname{cl}(G)$ into $Y$. Then $T$ is said to be $A$-proper with respect to a given approximation scheme in the sense of Definition 1 if for any sequence $\left\{n_{j}\right\}$ of positive integers with $n_{j} \rightarrow \infty$ and a corresponding sequence $\left\{x_{n_{j}}\right\}$ in $\operatorname{cl}(G)$ with each $x_{n_{j}}$ in $X_{n_{j}}$ such that $Q_{n_{j}} T x_{n_{j}}$ converges strongly in $Y$ to an element $y$, there exists an infinite subsequence $\left\{n_{j(k)}\right\}$ such that $x_{n_{j(k)}}$ converges strongly to $x$ in $X$ as $k \rightarrow \infty$ and $T(x)=y$.

The concept of $A$-proper mapping is a slight variant of the condition $(H)$ of Petryshyn [18], and both are modifications of the definition of $P$-compact mapping in Petryshyn [15], [16], and [17]. A sim- 
ilar definition has been given for strongly closed mappings by Pohojhayev [20].

DEFINITION 3. Let $T$ be an A-proper continuous mapping from $\mathrm{cl}(G)$ to $Y$ with respect to a given approximation scheme, and let a be a point of $Y-T(\operatorname{bdry}(G))$. Let $G_{n}=G \cap X_{n}$, and let $T_{n}=\left.Q_{n} T\right|_{a_{n}}$.

We define $\operatorname{Deg}(T, G, a)$, the degree of $T$ on $G$ over a with respect to the given scheme, as follows: Let $Z^{\prime}$ be the set of all integers (positive, negative, and zero) together with $\{+\infty\}$ and $\{-\infty\}$. Then $\operatorname{Deg}(T, G, a)$ is the subset of $Z^{\prime}$ given by:

(1). The integer $m$ lies in $\operatorname{Deg}(T, G, a)$ if there exists an infinite sequence of positive integers $n$ such that $\operatorname{deg}\left(T_{n}, G_{n}, Q_{n} a\right)$ is well-defined and equals $m$.

(2). $\pm \infty$ lies in $\operatorname{Deg}(T, G, a)$ if there exists an infinite sequence of integers $\left\{n_{j}\right\}$ with $n_{j} \rightarrow \infty$ such that $\operatorname{deg}\left(T_{n_{j}}, G_{n_{j}}, Q_{n_{j}} a\right)$ is well-defined for each $j$ and $\operatorname{deg}\left(T_{n_{j}}, G_{n_{j}}, Q_{n_{j}}\right.$ a) $\rightarrow \pm \infty$ as $j \rightarrow \infty$.

(The degree $\operatorname{deg}\left(T_{n}, G_{n}, Q_{n} a\right)$ used in Definition 3 is the classical Brouwer degree for mappings of oriented finite dimensional Euclidean spaces of the same dimension.)

Using the properties of the Brouwer degree and of $A$-proper maps, we obtain a direct and simple proof of the following theorem:

Theorem 1. Let $X$ and $Y$ be Banach spaces, $G$ a bounded open subset of $X, T$ an $A$-proper continuous mapping of $\operatorname{cl}(G)$ into $Y$ with respect to a given approximation scheme. Let a be a point of $Y-T(\operatorname{bdry}(G))$, and let $G_{n}=G \cap X_{n}, T_{n}=\left.Q_{n} T\right|_{G_{n}}$. Then:

(a) There exists an integer $n_{0} \geqq 1$ such that for $n \geqq n_{0}, Q_{n}$ a does not lie in $T_{n}$ (bdry $\left.G_{n}\right)$. Hence for such $n, \operatorname{deg}\left(T_{n}, G_{n}, Q_{n} a\right)$ is well-defined, and in particular, $\operatorname{Deg}(T, G, a)$ is a nonempty subset of $Z^{\prime}$.

(b) If $\operatorname{Deg}(T, G, a) \neq\{0\}$, there exists an element $x$ of $G$ such that $T(x)=a$.

(c) Let $T$ be a continuous mapping of $\operatorname{cl}(G) \times[0,1]$ into $Y$, and for each $t$, let $T_{t}(x)=T(x, t)$. Suppose that $T$ is uniformly continuous in $t$ on $[0,1]$, and that for each $t, T_{t}$ is $A$-proper with respect to a fixed approximation scheme from $X$ to $Y$. Then if a lies in $Y-T$ (bdry $(G)$ $\times[0,1])$, it follows that $\operatorname{Deg}\left(T_{t}, G, a\right)$ is independent of $t$ in $[0,1]$.

(d) Let $G=G_{1} \cup G_{2}$, and for $G^{\prime}=\left(G_{1} \cap G_{2}\right) \cup \operatorname{bdry}\left(G_{1}\right) \cup \operatorname{bdry}\left(G_{2}\right)$, suppose that $T\left(G^{\prime}\right)$ does not contain a. Then

$$
\operatorname{Deg}(T, G, a) \subset \operatorname{Deg}\left(T, G_{1}, a\right)+\operatorname{Deg}\left(T, G_{2}, a\right),
$$

with equality holding if either $\operatorname{Deg}\left(T, G_{1}, a\right)$ or $\operatorname{Deg}\left(T, G_{2}, a\right)$ is a singleton integer. (We use the convention that $\infty-\infty=Z^{\prime}$.)

Theorem 1 has as corollaries a number of interesting fixed point and mapping theorems for $A$-proper mappings. In the present discus- 
sion, we focus on an important special case for which the degree as defined in Definition 3 is single-valued.

Theorem 2. Let $X$ and $Y$ be Banach spaces, $G$ a bounded open subset of $X, T$ a continuous A-proper mapping of $\mathrm{cl}(G)$ into $Y, a \in Y$ $-T$ (bdry $G$ ). Suppose that we are given an approximation scheme in the sense of Definition 1 and $T=H+C$, where $C$ maps $\operatorname{cl}(G)$ into a relatively compact subset of $Y$ and $H$ maps $G$ homeomorphically onto an open subset $H(G)$ of $Y$, carrying $\mathrm{cl}(G)$ homeomorphically onto $\mathrm{cl}(H(G))$. Let $H_{n}=Q_{n} H, C_{n}=Q_{n} C, T_{n}=H_{n}+C_{n}$, with all these mappings restricted to $\operatorname{cl}\left(G_{n}\right)$ where $G_{n}=G \cap X_{n}$. Suppose that for each $n, H_{n}$ is an orientation preserving homeomorphism of $G_{n}$ into $Y_{n}$ and that the following condition holds:

(c) There exists a continuous, strictly increasing function $\alpha(r)$ for $r \geqq 0$ with $\alpha(0)=0$ such that for all $n$ and each pair $u$ and $v$ in $\mathrm{cl}\left(G_{n}\right)$,

$$
\left\|H_{n}(u)-H_{n}(v)\right\| \geqq \alpha(\|u-v\|) .
$$

Then there exists $n_{1} \geqq 1$ such that for $n \geqq n_{1}$,

$$
\operatorname{deg}\left(T_{n}, G_{n}, Q_{n} a\right)=\operatorname{deg}\left(I+C H^{-1}, H(G), a\right) .
$$

In particular, $\operatorname{Deg}(T, G, a)=\left\{\operatorname{deg}\left(I+C H^{-1}, H(G), a\right)\right\}$.

Corollary to TheORem 2. The conclusion of Theorem 2 holds in the case in which $X=Y$ and $T=H+C$, with $C$ compact and $H$ strongly accretive on $X$, i.e.

$$
(H u-H v, J(u-v)) \geqq c(\|u-v\|)
$$

for a continuous strictly increasing function $c(r)$ for $r \geqq 0$ with $c(0)=0$, and $J$ a duality mapping of $X$ into $X^{*}$ satisfying the conditions $(J u, u)$ $=\|J u\| \cdot\|u\|$ and $\|J u\|=\psi(\|u\|)$ for a continuous strictly increasing function $\psi$ with $\psi(0)=0$. We must assume in addition that the family of projections $Q_{n}$ has $\left\|Q_{n}\right\|=1$ for all $n$, and that either $X^{*}$ is uniformly convex or that $H$ is uniformly continuous on bounded subsets of $X$. (The latter case includes $H=I-U$, with $U$ a strict contraction.)

Proof of Theorem 2. Since $T$ is $A$-proper, we may assume that for all $n, Q_{n} a$ does not lie in $T_{n}$ (bdry $\left.G_{n}\right)$ so that $\operatorname{deg}\left(T_{n}, G_{n}, Q_{n} a\right)$ is well-defined. Since $H_{n}$ is an orientation preserving homeomorphism of $G_{n}$ in to $Y_{n}$, we have: $\operatorname{deg}\left(T_{n}, G_{n}, Q_{n} a\right)=\operatorname{deg}\left(I+C_{n} H_{n}^{-1}, H_{n}\left(G_{n}\right), Q_{n} a\right)$.

LEMMA 1. There exists $d>0$ such that for $n \geqq n_{2}$, with $n_{2}$ sufficiently large, $\left\|T_{n} u-Q_{n} a\right\| \geqq d$ for all $u$ in bdry $\left(G_{n}\right)$. Hence we may replace the compact map $C$ by $Q_{n} C$ and the point a by $Q_{m} a$ for a sufficiently large integer $m$ without changing either of the degrees in the conclusion of Theorem 2 for $n \geqq n_{2}$. 
Proof of Lemma 1. The second assertion follows from the first, along with standard properties of the degree. Suppose that the first assertion is false. Then there exists a sequence $\left\{n_{j}\right\}$ with $n_{j} \rightarrow \infty$ and a sequence $\left\{u_{n_{j}}\right\}$ with $u_{n_{j}} \in \operatorname{bdry}\left(G_{n_{j}}\right)$ such that $\left\|T_{n_{j}}\left(u_{n_{j}}\right)-Q_{n_{j}}(a)\right\|$ $\rightarrow 0$. Since $T$ is $A$-proper, we may assume that $u_{n j}$ converges strongly to $u$ in $X$ and that $T u=a$. Since each $u_{n_{j}}$ lies in bdry $(G), u$ must lie in bdry $(G)$. By hypothesis, there are no points in bdry $(G)$ for which $T u=a$.

q.e.d.

Lemma 2. Let $U$ be any neighborhood in $H(G)$ of the set $K_{1}=\{v \mid v$ $\in H(G), v=H(u)$, where $T(u)=a\}$. Let $S_{n}$ be the mapping of $H_{n}\left(G_{n}\right)$ into $Y$ given by $S_{n} u=Q_{n} C H_{n}^{-1}(u)$. Then there exists $n_{3} \geqq 1$ such that for $n \geqq n_{3}$, any point $v_{n}$ in $H_{n}\left(G_{n}\right)$ such that $\left(I+S_{n}\right)\left(v_{n}\right)=Q_{n} a$ must lie in the given neighborhood $U$.

Proof of Lemma 2. Suppose not. Then there will exist an infinite sequence $\left\{v_{n_{j}}\right\}$ with $v_{n_{j}} \in H_{n_{j}}\left(G_{n_{j}}\right)$ and $n_{j_{1}} \rightarrow \infty$ such that $v_{n_{j}}+S_{n_{j}} v_{n_{j}}$ $=Q_{n_{j}} a$ with each $v_{n_{j}}$ outside of $U$. Let $z_{n_{j}}=H_{n_{j}} v_{n_{j}}, z_{n_{j}} \in G_{n_{j}}$. Then

$$
T_{n_{j}}\left(z_{n_{j}}\right)=v_{n_{j}}+C_{n_{j}} H_{n_{j}}^{-1} v_{n_{j}}=Q_{n_{j}} a \text {. }
$$

Since $T$ is $A$-proper, we may pass to an infinite subsequence and assume that $z_{n_{j}}$ converges strongly to an element $z$ of $G$ for which $T z=a$. Hence $v_{n_{j}}=Q_{n_{j}} H\left(z_{n_{j}}\right)$ converges strongly to $H(z)$, which lies in $K_{1}$. Since $U$ is a neighborhood of $K_{1}$, this contradicts the fact that all the $v_{n_{j}}$ lie outside of $U$.

q.e.d.

Lemma 3. The set $K_{1}$ defined in Lemma 2 is compact, and there exists a neighborhood $U_{1}$ of $K_{1}$ and an integer $n_{4}$ such that for $n \geqq n_{4}, U_{1}$ is contained in $Q_{n}^{-1}\left(H_{n}\left(G_{n}\right)\right)$.

Proof of Lemma 3. The compactness of $K_{1}$ follows easily from the fact that $T$ is $A$-proper. Suppose the remainder of the assertion of Lemma 3 were not true. Then there would exist a sequence $\left\{y_{n_{j}}\right\}$ for $n_{j} \rightarrow \infty$ such that $\operatorname{dist}\left(y_{n_{j}}, K_{1}\right) \rightarrow 0$ for which $Q_{n_{j}} y_{n_{j}}$ does not lie in $Q_{n_{j}}\left(H\left(G \cap X_{n_{j}}\right)\right)$. Since $K_{1}$ is compact, we may assume that $y_{n_{j}}$ converges strongly as $j \rightarrow \infty$ to an element $y$ of $K_{1}$. Since $K_{1} \subset H(G)$, we may assume that each $y_{n_{j}}$ lies in $H(G)$ and form $w_{n_{j}}=H^{-1}\left(y_{n_{j}}\right)$. By the continuity of $H^{-1}, w_{n_{j}} \rightarrow w$ where $H(w)=y$, and $T(w)=a$.

For each $n$, we set $\epsilon_{n}=2 \operatorname{dist}\left(K_{1}, H\left(G_{n}\right)\right)$. Then $\epsilon_{n} \rightarrow 0$ as $n \rightarrow \infty$, and for each $y_{n_{j}}$ in the preceding paragraph, we may find $u_{n_{j}}$ in $G_{n_{j}}$ such that $\left\|y_{n_{j}}-H\left(u_{n_{j}}\right)\right\| \leqq \epsilon_{n_{j}}$. The hypothesis of Theorem 2 implies that there exists a constant $c>0$ such that $\left\|Q_{n}\right\| \leqq c$ for all $n$. Hence, $\left\|Q_{n_{j}} y_{n_{j}}-Q_{n_{j}} H\left(u_{n_{j}}\right)\right\| \leqq c \epsilon_{n_{j}}$. Since bdry $\left(H_{n}\left(G_{n}\right)\right)=H_{n}\left(\right.$ bdry $\left.G_{n}\right)$ for all $n$, it follows that $\operatorname{dist}\left(y_{n_{j}}, H_{n_{j}}\left(\right.\right.$ bdry $\left.\left.G_{n_{j}}\right)\right) \leqq c \epsilon_{n_{j}}$. Hence, we may find 
elements $v_{n_{j}}$ in bdry $G_{n_{j}}$ such that $\left\|Q_{n_{j}} y_{n_{j}}-Q_{n_{j}} H\left(v_{n_{j}}\right)\right\| \rightarrow 0$. Since $T$ is $A$-proper, so is $H=T-C$. Passing to an infinite subsequence, we may assume that $v_{n_{j}}$ converges strongly to an element $v$ of bdry $G$ for which $H(v)=y$, i.e. $v=w$ and $T(v)=a$. This is a contradiction, proving the lemma.

q.e.d.

Proof of Theorem 2 completed. By Lemma 1, we may assume that for $n \geqq n_{3}, Q_{n} C=C$ and $Q_{n} a=a$. We know that

$$
\operatorname{deg}\left(T_{n}, G_{n}, Q_{n} a\right)=\operatorname{deg}\left(I+C_{n} H_{n}^{-1}, H_{n}\left(G_{n}\right), Q_{n} a\right),
$$

and that

$d_{n}=\operatorname{deg}\left(I+C_{n} H_{n}^{-1}, H_{n}\left(G_{n}\right), Q_{n} a\right)=\operatorname{deg}\left(I+C H_{n}^{-1} Q_{n}, Q_{n}^{-1}\left(H_{n}\left(G_{n}\right)\right), a\right)$.

We wish to show this last degree to be equal to

$$
\delta=\operatorname{deg}\left(I+C H^{-1}, H(G), Q_{n} a\right)=\operatorname{deg}\left(I+C H^{-1}, H(G), a\right) .
$$

By Lemmas 2 and 3, we may choose a neighborhood $U$ of $K_{1}$ in $H(G)$ such that: $U \subset Q_{n}^{-1}\left(H_{n}\left(G_{n}\right)\right)$, while for any $v_{n}$ in $H_{n}\left(G_{n}\right)$ such that $\left(I+C_{n} H_{n}^{-1}\right)\left(v_{n}\right)=Q_{n} a$, we have $v_{n} \in U$. By Lemma 1 , we may assume that $a=Q_{m} a, C=Q_{m} C$. Hence for any $v$ in $Q_{n}^{-1}\left(H_{n}\left(G_{n}\right)\right)$ such that $v+C H_{n}^{-1} Q_{n} v=a$, we have $v \in Y_{n}$ and $Q_{n} v=v$ so that $v$ lies in $H_{n}\left(G_{n}\right)$. Thus,

$$
d_{n}=\operatorname{deg}\left(I+C H_{n}^{-1}, U \cap Y_{m}, a\right) ; \quad \delta=\operatorname{deg}\left(I+C H^{-1}, U \cap Y_{m}, a\right) .
$$

It suffices by the properties of the degree (e.g. [14]) to show that the mappings $\mathrm{CH}_{n}^{-1}$ converge uniformly to the mapping $\mathrm{CH}^{-1}$ on the compact set $K_{3}$ which is the closure of $U \cap Y_{m}$ in $H(G)$.

Let $u \in K_{3}$, and set $w=C H^{-1}(u), w_{n}=C H_{n}^{-1}(u), x=H^{-1}(u), x_{n}$ $=H_{n}^{-1}(u)$. The set $K_{4}=H^{-1}\left(K_{3}\right)$ is a compact subset of $G$, and hence $\operatorname{dist}\left(K_{4}, G_{n}\right)=2 \epsilon_{n} \rightarrow 0$. Therefore, we may find $y_{n}$ in $G_{n}$ such that $\left\|x-y_{n}\right\| \leqq \epsilon_{n}$. Since every continuous mapping is uniformly continuous at the points of a compact subset of its domain, there exists a sequence $\beta_{n} \rightarrow 0$ such that for all $u$ in $K_{3}$ and the corresponding point $x,\left\|H(x)-H\left(y_{n}\right)\right\| \leqq \beta_{n}$. Since $H(x)=u$ and $\left\|Q_{n}\right\| \leqq c$, we have

$$
\left\|H_{n}\left(x_{n}\right)-H_{n}\left(y_{n}\right)\right\|=\left\|u-Q_{n} H\left(y_{n}\right)\right\| \leqq\left\|u-Q_{n} u\right\|+\beta_{n} .
$$

Since $K_{3}$ is compact, there exists $\zeta_{n} \rightarrow 0$ such that $\left\|u-Q_{n} u\right\| \leqq \zeta_{n}$ for $u$ in $K_{3}$. Applying the condition (c) of the hypothesis of Theorem 2, we obtain

$$
\alpha\left(\left\|x_{n}-y_{n}\right\|\right) \leqq\left\|H_{n}\left(x_{n}\right)-H_{n}\left(y_{n}\right)\right\| \leqq \beta_{n}+\zeta_{n},
$$

so that 


$$
\left\|x-x_{n}\right\| \leqq\left\|x-y_{n}\right\|+\left\|y_{n}-x_{n}\right\| \leqq \epsilon_{n}+\alpha^{-1}\left(\beta_{n}+\zeta_{n}\right) \rightarrow 0,
$$

so that $H_{n}^{-1} u$ converge uniformly to $H^{-1} u$ on $K_{3}$. Finally, $C$ is continuous from $\operatorname{cl}(G)$ to $Y$ and hence uniformly continuous at points of the compact set $K_{4}$. Hence $C H_{n}^{-1}(u)$ converges uniformly to $\mathrm{CH}^{-1}(u)$ for $u$ in $K_{3}$.

q.e.d.

\section{BIBLIOGRAPHY}

1. F. E. Browder, Fixed point theorems for non-compact mappings in Hilbert space, Proc. Nat. Acad. Sci. U.S.A. 53 (1965), 1272-1276.

2. - Nonlinear operators in Banach spaces, Math. Ann. 162 (1966), 280-283.

3. - Fixed point theorems for nonlinear semicontractive mappings in Banach spaces, Arch. Rational Mech. Anal. 21 (1966), 259-269.

4. - Nonlinear accretive operators, Bull. Amer. Math. Soc. 73 (1967), 470-476.

5. - Nonlinear equations of evolution and nonlinear accretive mappings in Banach spaces, Bull. Amer. Math. Soc. 73 (1967), 867-874.

6. - Nonlinear mappings of nonexpansive and accretive type in Banach spaces, Bull. Amer. Math. Soc. 73 (1967), 875-881.

7. - Semicontractive and semiaccretive nonlinear mappings in Banach spaces, Bull. Amer. Math. Soc. 4 (1968), 600-665.

8. - Nonlinear operators and nonlinear equations of evolution in Banach spaces, Proc. Sympos. Nonlinear Functional Analysis, Amer. Math. Soc., April 1968 (to appear).

9. - Topology and nonlinear functional equations, Studia Math. (to appear).

10. F. E. Browder and D. G. de Figueiredo, J-monotone nonlinear operators in Banach spaces, Proc. Konin. Nederl. Akad. Wet. 28 (1966), 412-420.

11. F. E. Browder and R. Nussbaum, The topological degree for noncompact nonlinear mappings in Banach spaces, Bull. Amer. Math. Soc. 4 (1968), 671-676.

12. R. L. Frum-Ketkov, On mappings of the sphere in Banach space, Dokl. Akad. Nauk SSSR, 175 (1967), 1229-1231.=Soviet Math. Dokl. 8 (1967).

13. J. Leray and J. Schauder, Topologie et equations fonctionelles, Ann. Sci. Ecole Norm Sup. 51 (1934), 45-73.

14. M. Nagumo, Degree of mapping in convex linear topological spaces, Amer. J. Math. 73 (1951), 497-511.

15. W. V. Petryshyn, On a fixed point theorem for nonlinear P-compact operators in Banach spaces, Bull. Amer. Math. Soc. 72 (1966), 329-334.

16. - On nonlinear P-compact operators in Banach spaces with applications to constructive fixed-point theorems, J. Math. Anal. Appl. 15 (1966), 228-242.

17. - Further remarks on nonlinear P-compact operators in Banach spaces, J. Math. Anal. Appl. 16 (1966), 243-253.

18. - On the approximation solvability of nonlinear equations, Math. Ann. 177 (1968), 156-164.

19. W. V. Petryshyn and T. Tucker, On functional equations involving nonlinear generalized P-compact operators, Trans. Amer. Math. Soc. (to appear).

20. S. I. Pohojhayev, On the solvability of nonlinear equations with odd operators, Funct. Anal. Appl. 1 (1967), 66-73.

\section{University of Chicago and Rutgers UNIVERsity}

